\title{
Surface plasmon resonance enhanced ellipsometric analysis for monitoring of cobalt electrochemical reaction in solution
}

\author{
Zhenzhen Wanga,b, Wei Liu ${ }^{c}$, Chunxia Wang ${ }^{\mathrm{a}, *}$, Qiang Kan ${ }^{\mathrm{a}}$, She Chen ${ }^{\mathrm{c}}$, Hongda Chen ${ }^{\mathrm{a}}$ \\ a State Key Laboratory Integrated Optoelectronics, Institute of Semiconductors, Chinese Academy of Sciences, Beijing 100083, China \\ b Department of Physics, Tsinghua University, Beijing 100084, China \\ ${ }^{\mathrm{c}}$ Department of National Microgravity Laboratory, Institute of Mechanics, Chinese Academy of Sciences, Beijing 100083, China
}

\section{A R T I C L E I N F O}

\section{Article history:}

Received 27 September 2012

Received in revised form 17 January 2013

Accepted 20 January 2013

Available online 28 January 2013

\section{Keywords:}

Surface plasmon resonance

Ellipsometry

Ion selective electrode

Image contrast

\begin{abstract}
A B S T R A C T
Cobalt-based ion selective electrode (ISE) is one of the key methods for phosphate detection. In this paper, surface plasmon resonance (SPR) enhanced ellipsometric analysis method has been used to monitor the electrochemical reaction process at Co ISE sensor surface and the mass consumption of sensitive electrode membranes. An Au-Co bilayer film is designed as optical detection interface. In order to obtain detectable reflection intensity variation with Co thickness, Au film and Co film thickness are optimized at TM polarization state. According to the calculation results, $50 \mathrm{~nm}$ Au film is formed on glass substrate by electron beam evaporation. Then cobalt film with thickness of about $20 \mathrm{~nm}$ is grown on gold film by electrochemical deposition method. Ellipsometric measurements were performed at SPR angle $59^{\circ}$. The consuming process of $\mathrm{Co}$ film reacting with dissolved oxygen in deionized water has been obtained by monitoring the change of reflection intensity.
\end{abstract}

(C) 2013 Elsevier B.V. All rights reserved.

\section{Introduction}

Ellipsometry is one of the most important methods to determine the surface layer state according to the change in the polarization state of light beam reflected from the sample. Compared to other methods widely used in the study of surface layer such as Raman scattering spectroscopy [1] and atomic force microscopy [2], ellipsometry is a fundamentally non-invasive technique for film analysis with high sensitivity and real time characteristics [3-9]. Recently, surface plasmon resonance (SPR) enhanced ellipsometry detection has attracted people's interests and the detection sensitivity has been greatly increased [8,9]. Surface plasmon polaritons (SPPs) are electromagnetic surface mode associated with collective electron oscillation propagating along the interface between a metal and a dielectric [10]. In light of its localization and amplitude enhancement $[11,12]$, SPR can be used in ellipsometric method to enhance the measured light signals. SPR enhanced ellipsometric method has been recognized as a powerful tool due to its high sensitivity for detection of thin organic layer and reaction kinetics on solid substrate [8,9]. Moirangthem et al. have adopted SPR ellipsometry to study basic properties of bimolecular interaction which yields very high sensitivity [9].

\footnotetext{
* Corresponding author. Tel.: +86 10 82304531; fax: +86 1082304416 .

E-mail address: cxwang@semi.ac.cn (C. Wang).
}

The detection of phosphorus concentration levels is important in many areas, such as environmental monitoring, clinical chemistry, and biomedical research. Cobalt-based ion selective electrode (ISE) is a successful option for phosphate detection [13,14]. Various microelectrochemical sensors integrated with working electrode, reference electrode and microfluidics channels are developed based on microelectromechanical systems technology $[14,15]$. The ISE sensor based on cobalt $(\mathrm{Co})$ is a normally used method for phosphate detection and has demonstrated a lot of promise. The sensing mechanism is premised on Co dissolution at electrode surface and formation of oxide film $[15,16]$. When phosphate ions are present in solution, they will react with the cobalt oxide ( $\mathrm{CoO})$ film and form cobalt phosphate. A linear potential response can be expected to logarithmic phosphate concentration at fixed concentration level of dissolved oxygen [17]. The main drawback of using thin Co film as microelectrode membrane includes a limited lifetime due to Co dissolution. The questions that need to be addressed relate to how the mass consume of Co sensitive membrane in phosphate solution or even in water can be monitored. Thus far, there are few research reports that involve the evaluating of working membrane sensing duration and stabilities.

In order to monitor the mass consumption of sensitive electrode membranes in reaction with dissolved oxygen, SPR enhanced ellipsometric analysis has been proposed in this paper. We have designed an $\mathrm{Au}-\mathrm{Co}$ bilayer film as optical detection interface. In order to obtain detectable reflection intensity variation with Co thickness, we have optimized two factors: Au film and Co film 
(a)

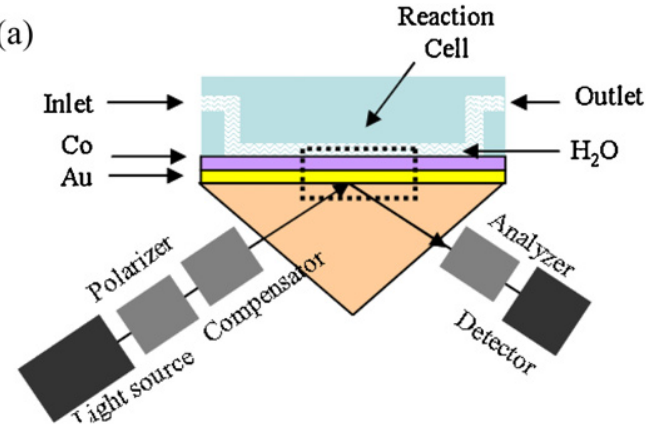

(b)

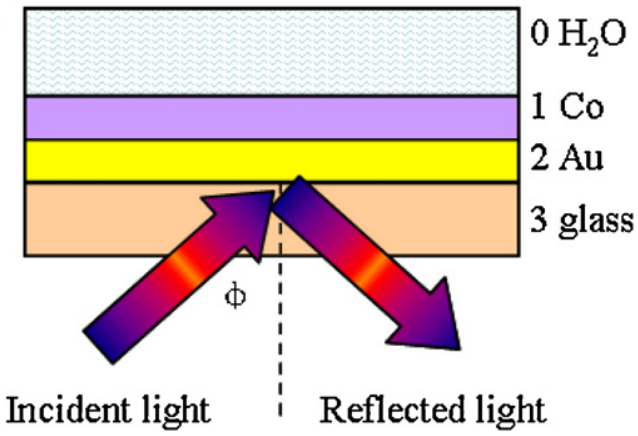

Fig. 1. (a) The schematic diagram of the SPR enhanced ellipsometric analysis system and (b) optical detection interface of Au-Co bilayer.

thickness at TM polarization state incidence. Finally, the real-time reaction process and the mass consumption rate of Co electrode membrane have been obtained by SPR enhanced ellipsometry.

\section{Optical design}

\subsection{Theoretical model}

The SPR enhanced ellipsometric analysis system is shown in Fig. 1(a). In our model, an Au-Co bilayer film is introduced as optical detection interface, with glass prism substrate and deionized water as surrounding medium. A polydimethylsiloxane (PDMS) reaction cell is tightly attached to the sample to form the micro-channel for $\mathrm{Co}$ and dissolved oxygen reaction in solution. The Co film could react with water molecules and dissolved oxygen in the form of following chemical reaction equations:

$2 \mathrm{Co}+2 \mathrm{H}_{2} \mathrm{O} \Leftrightarrow 2 \mathrm{CoO}+4 \mathrm{H}^{+}+4 \mathrm{e}^{-}$

$\mathrm{O}_{2}+4 \mathrm{H}^{+}+4 \mathrm{e}^{-} \Leftrightarrow 2 \mathrm{H}_{2} \mathrm{O}$

$2 \mathrm{Co}+\mathrm{O}_{2} \Leftrightarrow 2 \mathrm{CoO}$

A polarized TM incident light beam goes through a prism and reaches the Au layer at a certain angle. SPR will appear when the momentum mismatch between the wave vector of SPPs and that of incident light is overcome. SPR is widely employed for label-free biosensing of analyte molecules that bind on the surface of metallic thin film [18-20]. This will change the oscillation of surface plasmons which in turn affects the modulation of light reflection or transmission signals. The modulation includes the angular distributions, wavelength, intensity, phase, and polarization changes [21]. In our model, when the thickness of thin Co film on Au film surface changes, the oscillation of surface plasmons is affected, and as a result, the reflected light intensity changes. The intensity modulation depth of SPR on the gold layer surface depends on the angle of incidence and the thickness of the metals (Au and Co). Our goals are finding the SPR resonance angle and optimizing the thickness of $\mathrm{Au}$ and $\mathrm{Co}$ at TM polarization state to get the detectable intensity signal varieties and maximized sensitivity.

A stratified structure consisting of a stack of $0, \ldots, n(1 \leq n \leq 3$, integer) parallel layers shown in Fig. 1(b) has been taken for the theoretical simulation. The number $0,1,2,3$ represent superstrate (water), Co layer, Au layer, and substrate (glass), respectively. According to the Fresnel formula, the reflection and transmission coefficients $(r$ and $t)$ at $p$ and $s$ polarizations in medium $N_{i}$ on an adjacent medium with refractive index $N_{i-1}$ can be expressed as:

$$
\begin{aligned}
r_{(i-1, i) p} & =\frac{N_{i} \cos \varphi_{i-1}-N_{i-1} \cos \varphi_{i}}{N_{i} \cos \varphi_{i-1}+N_{i-1} \cos \varphi_{i}}, \\
r_{(i-1, i) s} & =\frac{N_{i-1} \cos \varphi_{i-1}-N_{i} \cos \varphi_{i}}{N_{i-1} \cos \varphi_{i-1}+N_{i} \cos \varphi_{i}}, \\
t_{(i-1, i) p} & =\frac{2 N_{i-1} \cos \varphi_{i-1}}{N_{i} \cos \varphi_{i-1}+N_{i-1} \cos \varphi_{i}}, \\
t_{(i-1, i) s} & =\frac{2 N_{i-1} \cos \varphi_{i-1}}{N_{i-1} \cos \varphi_{i-1}+N_{i} \cos \varphi_{i}},
\end{aligned}
$$

where $\varphi_{i}$ is the angle of incidence in the $i$ th layer.

For the water-Co-Au multilayer system, from the calculation of complex amplitude of multiple beam interference, the total reflection coefficient of the system $r_{(0,1,2) p, s}$ could be iteratively deduced by the following equations:

$r_{(0,1,2) p, s}=\frac{r_{(0,1) p, s}+r_{(1,2) p, s} e^{-i 2 \delta_{1}}}{1+r_{(0,1) p, s} r_{(1,2) p, s} e^{-i 2 \delta_{1}}}$,

For the whole system as shown in Fig. 1(b), the final total complex amplitude reflection coefficient of the system $r_{(0,1,2,3) p, s}$ is

$r_{(0,1,2,3) p, s}=\frac{r_{(0,1,2) p, s}+r_{(2,3) p, s} e^{-i 2 \delta_{2}}}{1+r_{(0,1,2) p, s} r_{(2,3) p, s} e^{-i 2 \delta_{2}}}$,

The phase difference between the two interfaces of the $i$ th layer $\delta_{i}=\left(2 \pi N_{i} d_{i} \cos \varphi_{i}\right) / \lambda(i=1,2)$ is determined by the refractive index $N_{i}$, the thickness $d_{i}$, the incident angle $\varphi_{i}$, and the wavelength $\lambda$. In the following, we use $r_{p, s}$ instead of $r_{(0,1,2,3) p, s}$ for simplicity. We set $R_{p}$ and $R_{s}$ as the $p$ and $s$ component reflectivity, which is the ratio of the reflection intensity to incident light intensity. They are given by

$R_{p}=\left|r_{p}\right|^{2}, \quad R_{s}=\left|r_{s}\right|^{2}$

From the above derivation, we can see the total reflectivity $R_{p}$ and $R_{s}$ are determined by the refractive index $N_{i}(0 \leq i \leq 3)$, the thickness $d_{i}(i=1,2)$, incident angle $\varphi$ and wavelength $\lambda$. In the following calculation and experiment, in order to excite SPPs, the incident light is set as $p$-polarized. The wavelength $\lambda$ is set as $633 \mathrm{~nm} . N_{i}(0 \leq i \leq 3)$ is kept constant while the structure materials are fixed. Therefore, the reflectivity is finally determined by the thickness of Au and Co layers and angle of incidence. Suppose the reflection intensity of stack of parallel layers with and without $\mathrm{Co}$ film as $I\left(\varphi, d_{\mathrm{Au}}, d_{\mathrm{Co}}\right)$ and $I\left(\varphi, d_{\mathrm{Au}}, 0\right)$, respectively. In the experiment, the recording signal in the experiment is real-time reflection intensity. It helps to improve system sensitivity by increase the contrast of reflection intensity. So in the optimization calculation, we define image contrast as contrast of the reflection intensity to optimize the thickness of metal films. It could be described as [8]

$\Delta I=\frac{I\left(\varphi, d_{\mathrm{Au}}, d_{\mathrm{Co}}\right)-I\left(\varphi, d_{\mathrm{Au}}, 0\right)}{I_{i}}=R\left(\varphi, d_{\mathrm{Au}}, d_{\mathrm{Co}}\right)-R\left(\varphi, d_{\mathrm{Au}}, 0\right)$

where $I_{i}$ is the intensity of the input light, $R\left(\varphi, d_{\mathrm{Au}}, d_{\mathrm{CO}_{\mathrm{O}}}\right)$ and $R\left(\varphi, d_{\mathrm{Au}}, 0\right)$ represent the $\mathrm{p}$-polarized reflectivity while the Co layer thickness is $d_{\text {Co }}$ and 0 respectively at fixed incident angle $\varphi$ and Au layer thickness $d_{\mathrm{Au}}$. The image contrast is determined by the 

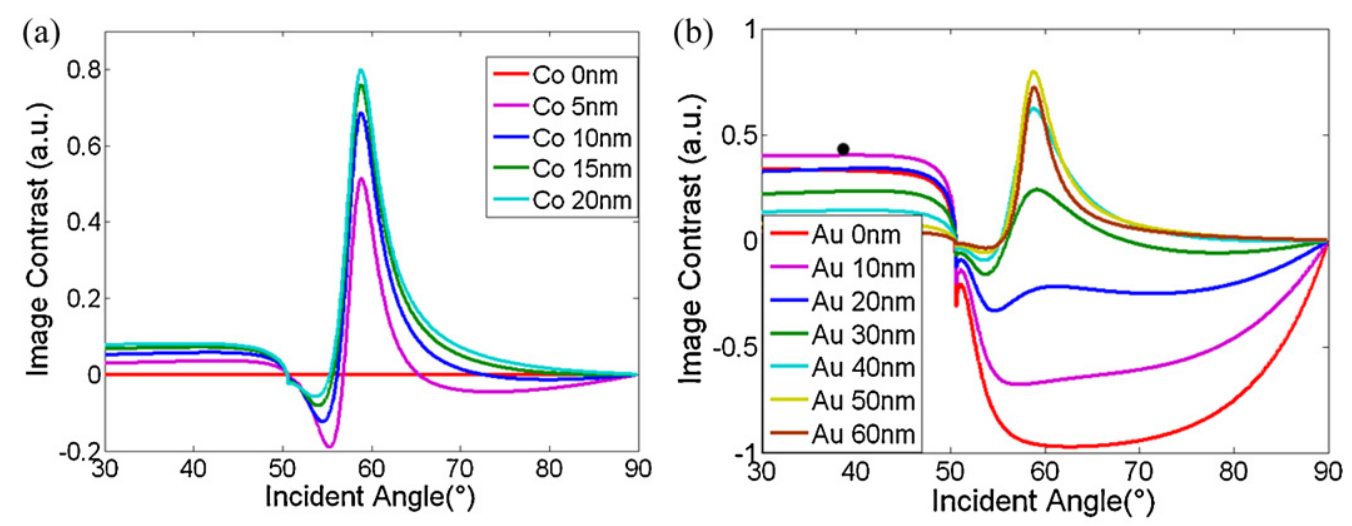

(c)

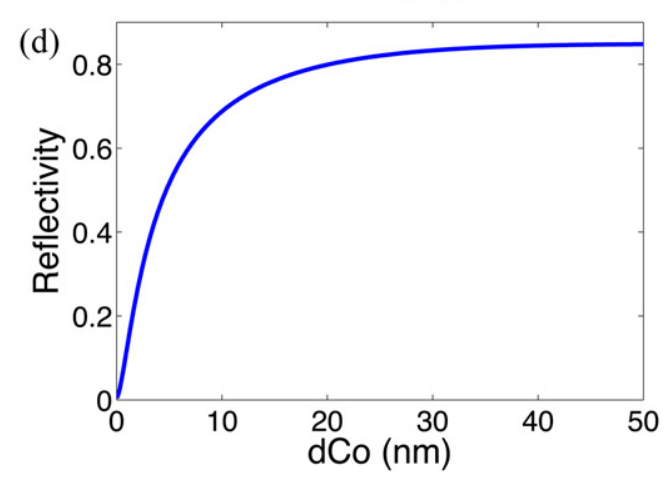

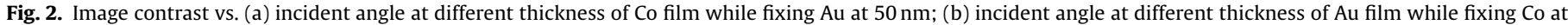
$20 \mathrm{~nm}$; reflectivity vs. the thickness of (c) Au and Co films at SPR angle $59^{\circ}$ and (d) Co film at SPR angle $59^{\circ}$ when Au is $50 \mathrm{~nm}$ thick.

reflection intensity difference. For Co film with a fixed thickness, the bigger the image contrast is the more sensitive the detection is.

\subsection{Configuration optimization of bilayer metal film}

Firstly, we fix the thickness of Au film at $50 \mathrm{~nm}$. The relationship between image contrast and incident angle at different thickness of Co film, changed from 0 to $20 \mathrm{~nm}$ with gradient of $5 \mathrm{~nm}$, is shown in Fig. 2(a). There exists a minimum image contrast value at about $55^{\circ}$ incident angle and a maximum at about $59^{\circ}$ incident angle where surface plasmon resonance takes place. At $55^{\circ}$ incident angle, image contrast varies non-monotonically with Co film thickness. With the increase of Co thickness, the image contrast increases first and gets its maximum when Co film is about $5 \mathrm{~nm}$, and then decreases. At $59^{\circ}$ incident angle, image contrast increases with the increasing of Co film thickness monotonically. In Fig. 2(b), we fix the thickness of Co film at $20 \mathrm{~nm}$ and obtain the relationship between image contrast and incident angle at different thickness of Au film which varies from 0 to $60 \mathrm{~nm}$ with gradient of $10 \mathrm{~nm}$. The image contrast gets its maximum when $\mathrm{Au}$ film is $50 \mathrm{~nm}$ at $59^{\circ}$ incident angle.

Fig. 2(c) shows the relationship between reflectivity and the thickness of Au and Co bilayer while the incident angle is set at the SPPs resonance angle $59^{\circ}$. There always exists a maximized extinction point at proper $\mathrm{Au}-\mathrm{Co}$ film thickness, which corresponds to the strongest coupling between SPPs and the evanescent wave of incident field. In order to monitor the consuming process of Co film, we chose $50 \mathrm{~nm}$ bottom Au seed layer in experiment. As shown in Fig. 2(d), if the thickness of Au film is fixed, when Co layer thickness is decreased from $50 \mathrm{~nm}$ to zero, the reflectivity also decreases monotonically, which yields the quantitative measurement of Co layer thickness. We set initial Co layer thickness as $20 \mathrm{~nm}$ in experiment.

\section{Chip fabrication}

Firstly, the Au seed layer was formed on glass substrate by electron beam evaporation (EBE). Then electrochemical deposition was taken to form the Co film. Electrocircuit connection diagram of the electroplating is shown in Fig. 3. The working electrode was prepared from high purity Co rod, which had been rinsed in $20 \%$ hydrochloric acid $(\mathrm{HCl})$ solution for $15 \mathrm{~min}$ to remove the CoO layer on the surface and the reference electrode was the glass substrate coated with $\mathrm{Au}$ film. The electrolyte was prepared by dissolve $33 \mathrm{~g}$ cobalt sulfate $\left(\mathrm{CoSO}_{4}\right)$ and $3 \mathrm{~g}$ boric acid $\left(\mathrm{H}_{3} \mathrm{BO}_{3}\right)$ in $100 \mathrm{~mL}$ deionized water.

According to Faraday's law, the mass $m$ of the Co film coated on the Au film is determined by the molar mass $M$ of Co, the current $I$ in the circuit, and the electroplating time $t$, shown in the following formula:

$m=M \frac{I t}{F}$

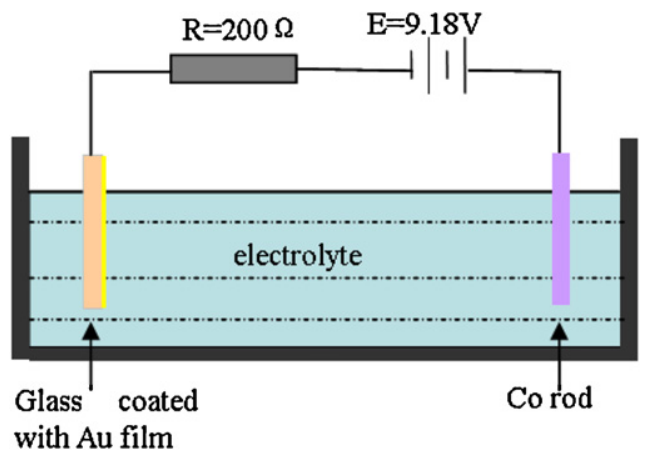

Fig. 3. Electrocircuit connection diagram of the electroplating process. 
(a)

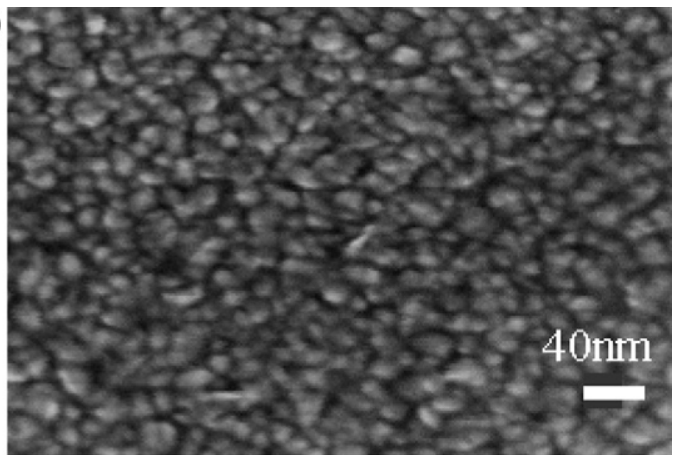

(b)

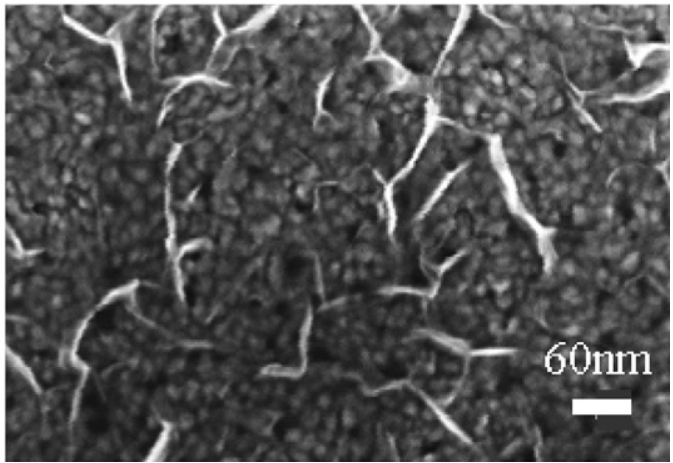

Fig. 4. SEM graphs of (a) Au film formed by EBE method and (b) Co film produced by electrodeposition.

Here, $F$ is Faraday constant. Then replace $m$ and $I$ with $r \cdot s \cdot t$ and $D \cdot s$ respectively where $r$ is density of Co, $s$ and $d$ are the area and thickness of the Co film electroplated, $D$ is current density, and the following equation can be obtained:

$d=M \frac{D t}{r F}$

From the above expression we can see the thickness of Co film electroplated on Au film is substantially determined by $D$ and $t$. So in a fixed electroplating circuit, we could change voltage $E$ and electroplating time $t$ to get the right thickness of Co film. In this paper, we fixed $E$ at $9.18 \mathrm{~V}$. After $4.5 \mathrm{~s}$, a thin Co film was obtained. The thickness of the film was measured by profiler and the average thickness value was about $27 \mathrm{~nm}$ from the data at different points. The SEM graphs are shown in Fig. 4. Co film (Fig. 4(b)) shows good uniformity as well as Au seed film (Fig. 4(a)).

\section{Results and discussion}

Fig. 5(a) shows the experimental setup for real-time ellipsometry reflection signal measurement. There are one inlet and one outlet for the reaction solutions connected with the reaction cell. The PDMS reaction cell is tightly attached to the chip to form the micro-channel for Co and dissolved oxygen reaction in solution. Then a glass prism is attached to the back side of the chip for optical signal detection. Optical measurements were performed at incident angle $59^{\circ}$. The real-time ellipsometry reflection signal was recorded when deionized water was injected into the reaction cell by a peristaltic pump with the rate of $50 \mu \mathrm{L} / \mathrm{min}$. The reactions (as shown in chemical reaction Eqs. (1)-(3)) between Co film, water molecules and dissolved oxygen took place rapidly in solution. As a small amount of cobalt oxide ( $\mathrm{CoO}$ ) could be dissolved in water (solubility in water: $33 \mathrm{~g} / \mathrm{L}$ [22]) at room temperature and atmospheric pressure, then what can be observed was that the Co film became gradually thinner until the film was almost exhausted. When the deionized water was continuously injected into

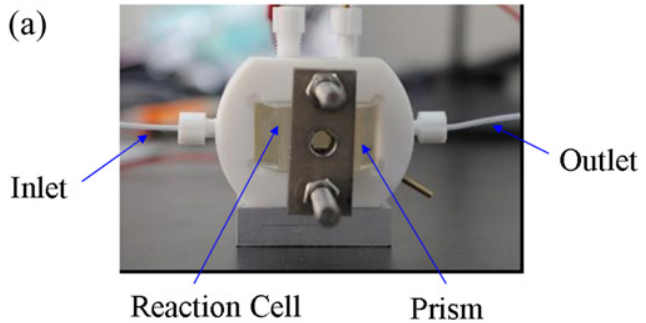

(The chip is located between the reaction cell and prism )

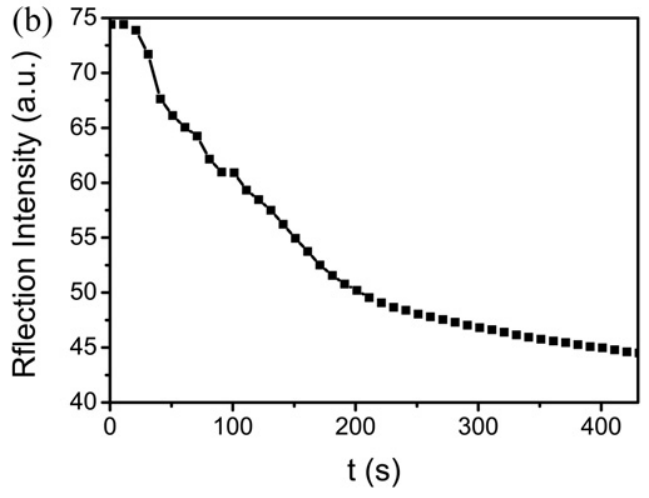

Fig. 5. (a) Experimental setup for real-time ellipsometry reflection signal measurement and (b) the time-evolved ellipsometric reflection intensity in the reaction process of Co film and dissolved oxygen at microfluidic channels.

micro-channel slowly, the reaction cell was fulfilled with water and the concentration of dissolved oxygen near the Co film surface was kept almost fixed, so we can anticipate that the consumption speed of Co was kept nearly constant with time. With the consumption of Co film, the reflection intensity at SPPs resonant angle changes correspondingly, shown as the decrease of reflection intensity in the ellipsometric analysis system. The time-evolved reflection intensity signal is shown in Fig. 5(b). After 430 s, the detected reflectivity signal became stable which implied that the $27 \mathrm{~nm}$ Co film was mostly depleted.

It should be noted that there is a gap for reflection intensity between simulation and experiment. In simulation, the reflection intensity is zero which implies that the coupling efficiency of incident power to SPPs is near $100 \%$. In experiment, Au film formed by electron beam evaporation has a rough surface with Au mean particle size of about $10 \mathrm{~nm}$. The rough surface would affect the coupling efficiency of incident power to SPPs. Also, the film quality of $\mathrm{Au}$ and $\mathrm{Co}$ is not ideal as that taken in calculation. Meanwhile, this nonuniform metal film could make actual SPR resonance angle deviating a little from $59^{\circ}$. This is the main reason why there is still some light reflected as $d_{\text {Co }}$ equals to zero. However, we can still conclude whether $\mathrm{Co}$ is exhausted as the reflection intensity curve becomes flattened in later reaction stage. Fig. 6(a) and (b) shows microscopy image of the chip before and after the reaction. Comparing the two images, the Co film in reaction cell denoted as region 3 was mostly consumed, which could also be proved by the energy dispersion X-ray (EDX) spectra shown in Fig. 6(c). In the reference region, there are characteristic spectral lines of both $\mathrm{Au}$, Co and substrate elements, while in the reaction cell region there are only characteristic spectral lines of $\mathrm{Au}$ and substrate elements. The EDX spectrum of the reaction cell is almost the same as that of Au seed layer coated on glass substrate. The average Co film mass consumption rate can be evaluated as $6.28 \mathrm{~nm} / 100 \mathrm{~s}$ based on expression (8). Our experiment measurement indicate that SPR 
(a)

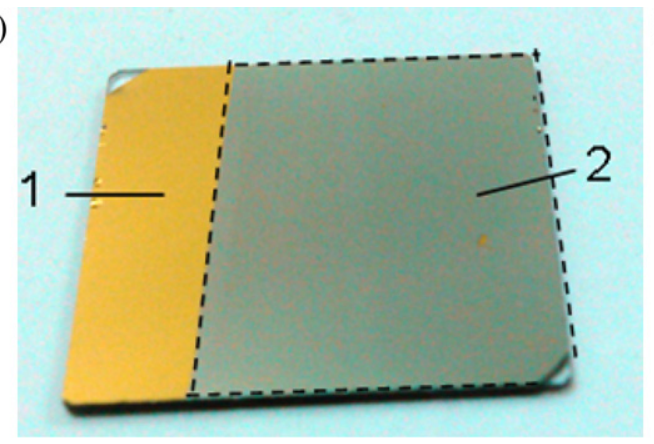

(b)

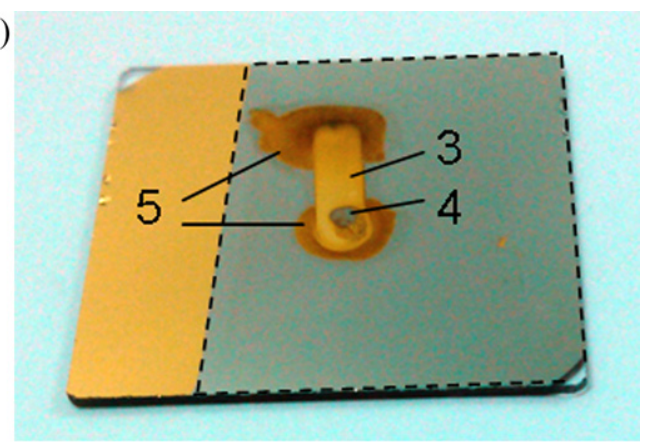

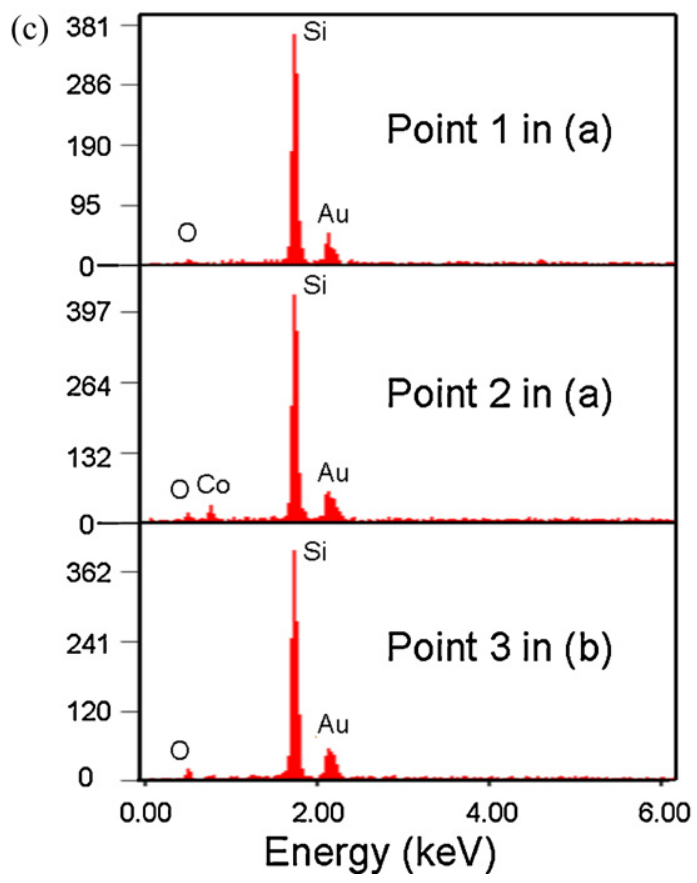

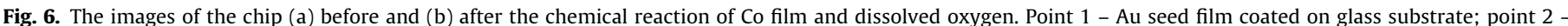

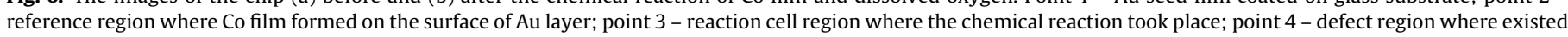

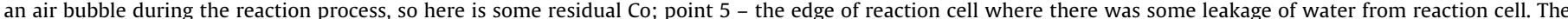
reversible bonding between PDMS and Au is not so tight that some of Co film was reacted with leaked water and (c) EDX spectra of points 1,2 and 3 on the chip surface.

enhanced ellipsometric analysis has good sensitivity and real-time detection capability in electrochemical reaction monitoring.

\section{Conclusion}

SPR enhanced ellipsometry has been introduced to detect the reaction between Co film and dissolved oxygen in water solution. In order to get detectable reflection intensity variation with Co thickness, the Au-Co bilayer film thickness has been optimized theoretically. At proper Au layer and Co layer configuration, the reflection intensity decreases monotonically with the reduction of Co film mass. The consuming process of Co film in deionized water has been well monitored by measuring reflection intensity. Our experiment results show SPR enhanced ellipsometry has good sensitivity in detection of electrochemical reaction on the surface of thin metal film.

\section{Acknowledgements}

This work was supported by the National Basic Research of China (nos. 2009CB320300, 2010CB934104, 2011CB933203 and
2011CB933102) and the Nation Natural Foundation of China (Grant nos. 61036009 and 60978067).

\section{References}

[1] G. Mcnay, D. Eustace, W.E. Smith, Surface-enhanced Raman scattering (SERS) and surface-enhanced resonance Raman scattering (SERRS): a review of applications, Applied Spectroscopy 65 (2011) 825-837.

[2] P.L.T.M. Frederix, P.D. Bossart, A. Engel, Atomic force microscopy of biological membranes, Biophysical Journal 96 (2009) 329-338.

[3] K. Watanabe, N. Matsuki, H. Fujiwara, Ellipsometry characterization of hydrogenated amorphous silicon layers formed on textured crystalline silicon substrates, Applied Physics Express 3 (2010) 116604.

[4] M.C. Howland, A.W. Szmodis, B. Sanii, A.N. Parikh, Characterization of physical properties of supported phospholipids membranes using imaging ellipsometry at optical wavelengths, Biophysical Journal 92 (2007) 1306-1317.

[5] Q.X. Zhang, W.S. Wei, F.P. Ruan, Ellipsometric analysis and optical absorption characterization of gallium phosphide nanoparticulate thin film, Chinese Physics B 20 (2011) 047802.

[6] X.Y. Gao, H.L. Feng, J.M. Ma, Z.Y.Zhang, Spectroscopic ellipsometric study of the optical properties of $\mathrm{Ag}_{2} \mathrm{O}$ film prepared by direct-current magnetron reactive sputtering, Chinese Physics B 19 (2010) 090701.

[7] V.A. Kotenev, A.Y. Tsvadze, Laser-ellipsometric monitoring of corrosive attack Protection of Metals and Physical Chemistry of Surfaces 45 (2009) 472-486.

[8] L. Liu, Y. Niu, S. Chen, Y.H. Meng, H.W. Ma, G. Jin, Optimization of evanescent wave imaging for the visualization of protein absorption layer, Science China 53 (2010) 1805-1810. 
[9] R.S. Moirangthem, Y.C. Chang, S.H. Hsu, P.K. Wei, Surface plasmon resonance ellipsomtry based sensor for studying biomolecular interaction, Biosensors and Bioelectronics 25 (2010) 2633-2638.

[10] W.L. Barnes, A. Dereux, T.W. Ebbesen, Surface plasmon subwavelength optics, Nature 424 (2003) 824-830.

[11] X.L. Zhong, Z.Y. Li, Plasmon enhanced light amplification in metal-insulator-metal waveguides with gain, Journal of Optics 14 (2012) 055002.

[12] R. Xu, X.D. Wang, W. Liu, X.N. Xu, Y.Q. Li, A. Ji, F.H. Yang, J.M. Li, Chinese Physics B 21 (2012) 025202.

[13] D. Xiao, H.Y. Yuan, J. Li, R.Q. Yu, Surface-modified cobalt-based sensor as a phosphate-sensitive electrode, Analytical Chemistry 67 (1995) 288291.

[14] Z. Zou, J. Han, A. Jang, P.L. Bishop, C.H. Ahn, A disposable on-chip phosphate sensor with planar cobalt microelectrodes on polymer substrate, Biosensors and Bioelectronics 22 (2007) 1902-1907.

[15] J.H. Lee, W.H. Lee, P.L. Bshop, I. Papautsky, A cobalt-coated needle-type microelectrode array sensor for in situ monitoring of phosphate, Journal of Micromechanics and Microengineering 19 (2009) 025022.

[16] R.K. Meruva, M.E. Meyerhoff, Mixed potential response mechanism of cobalt electrodes toward inorganic phosphate, Analytical Chemistry 68 (1996) 2022-2026.

[17] Z.L. Chen, P. Grierson, M.A. Adama, Direct determination of phosphate in soil extracts by potentiometric flow injection using a cobalt wire electrode, Analytica Chimica Acta 363 (1998) 191-197.

[18] H.Y. Zhang, L.Q. Yang, L. Meng, J.C. Nie, T.Y. Ning, W.M. Liu, J.Y. Sun, P.F. Wang, Real time detection of antibody-antigen interaction using a laser scanning confocal imaging-surface plasmon resonance system, Chinese Physics B 21 (2012) 020601.

[19] S. Scarano, M. Mascini, A.P.F. Turner, M. Minunni, Surface plasmon resonance imaging for affinity-based biosensors, Biosensors and Bioelectronics 25 (2010) 957-966.

[20] B. Buchele, W. Zugmaier, O. Lunov, T. Syrovets, I. Merfort, T. Simmet, Surface plasmon resonance analysis of nuclear factor- $\mathrm{B}$ protein interactions with the sesquiterpene lactone helenalin, Analytical Biochemistry 401 (2010) 30-37.

[21] A. Dhawan, M. Canva, T. Vo-Dinh, Narrow groove plasmonic nano-gratings for surface plasmon resonance sensing, Optics Express 19 (2011) 787813.

[22] http://zh.wikipedia.org/wiki/\%E6\%B0\%A7\%E5\%8C\%96\%E4\%BA\%9A\%E9\%92\%B4

\section{Biographies}

Zhenzhen Wang is currently working as a PhD student in the Institute of Semiconductors, Chinese Academy of Sciences. Her major is microelectronics and optoelectronics. Her current scientist interests are surface plasmon resonance enhanced biosensing for environment monitoring.

Wei Liu is a PhD candidate in the Chinese Academy of Sciences. He works in the Institute of Mechanics. His current scientist interest is dissolved oxygen measurement combined by total internal reflection ellipsometric technique and electrochemistry method.

Chunxia Wang received her MSc and PhD degrees from Wuhan University in 2002 and 2007, respectively. She is now a research scientist at the Institute of Semiconductors, the Chinese Academic of Science in the field of nanophotonics and micro \& nano-devices. Her main interests are smart biosensor realization for environment monitoring and novel sensing mechanism studies.

Qiang Kan received his bachelor degree in physics from the Harbin Institute of Technology in 2000 and his $\mathrm{PhD}$ degree in microelectronics and solid state electronics from Institute of Semiconductors, Chinese Academy of Science in 2005. He is currently working in State Key Laboratory Integrated Optoelectronics, Institute of Semiconductors, Chinese Academy of Sciences. His main interests are the surface plasmon resonance sensor and semiconductor lasers.

She Chen is currently a senior engineer in the Institute of Mechanics (IMECH) Chinese Academy of Sciences. He studied physics at the Beijing University of Technology before receiving his diploma in 1994. He received his MSc degree in genera mechanics and mechanics foundation from the IMECH, CAS in 2005 with a thesis on numerical simulation of adaptive optics system. Since July of 1994 he has been assistant researcher, later senior engineer at IMECH. His current scientific interests are near-field optics and ellipsometry.

Hongda Chen received his MSc and PhD degrees from Tianjin University in 1990 and 1996, respectively. 1997-2001, he was associate professor at Institute of Semiconductors, Chinese Academy of Sciences (ISCAS). Since 2001, he has been full professor at State Key Laboratory on Integrated Optoelectronics of ISCAS. His main research interests are fundamentals of semiconductor devices, optoelectronic devices and integration technology, very short reach (VSR) module and system, silicon based device $\&$ integrated circuits for biomedical applications. 